\title{
One-loop Euclidean Einstein-Weyl gravity in de Sitter universe
}

\author{
Guido Cognola $^{(a)}$ *, Emilio Elizalde ${ }^{(b)}$ 丹, Sergio Zerbini ${ }^{(a)}$ 团 \\ (a) Dipartimento di Fisica, Università di Trento \\ and Istituto Nazionale di Fisica Nucleare, \\ Gruppo Collegato di Trento, Italia \\ (b) Consejo Superior de Investigaciones Científicas ICE/CSIC-IEEC, \\ Campus UAB, Facultat de Ciències, \\ Torre C5-Parell-2a pl, 08193 Bellaterra (Barcelona) Spain
}

\begin{abstract}
By making use of the background field method, the one-loop quantization for Euclidean EinsteinWeyl quadratic gravity model on the de Sitter universe is investigated. Using generalized zeta function regularization, the on-shell and off-shell one-loop effective actions are explicitly obtained and one-loop renormalizability, as well as the corresponding one-loop renormalization group equations, are discussed. The so called critical gravity is also considered.
\end{abstract}

PACS numbers: $\quad$ 04.50.Kd, 95.30.Sf, 11.10.Hi

*e-mail: cognola@science.unitn.it

${ }^{\dagger}$ e-mail: elizalde@ieec.uab.es

${ }^{\ddagger} \mathrm{e}$-mail: zerbini@science.unitn.it 


\section{Introduction}

Recent astrophysical data indicate that our universe is currently in a phase of accelerated expansion. The physical origin of this acceleration is not completely understood and the related issue is commonly called the dark energy problem.

Several possible explanations have been proposed in the literature. One of them is based on the use of modified gravitational models, the simplest one consisting in the inclusion of a small and positive cosmological constant. Such model works quite well, according to the most recent data, but nevertheless it has some drawbacks (see, for example [1, 2, 3, 4, and reference therein).

Roughly speaking, the idea underlying modified gravity models is that the Einstein-Hilbert action gives only an approximate low energy contribution to gravitation and additional terms depending on the quadratic curvature invariants should necessarily be included. This idea is quite old since it was already contained in the seminal paper [5], where quadratic terms in the curvature, justified by quantum effects, were added to the Einstein-Hilbert Lagrangian (for a review, see [6]). The important recent finding is that the inclusion of suitable higher order contributions may realize not only the actual accelerated expansion, but also the early time inflation epoch [7]. Within this context, the de Sitter (dS) space-time plays a fundamental role, being able to provide an acceleration at different stages in the cosmological set up.

In previous papers [8, 9, 10, 11, $f(R)$ gravity models and a non local Gauss-Bonnet gravity model at one-loop level in a de Sitter background have been investigated. A similar program for the case of pure Einstein gravity was initiated in Refs. [12, 13, 14] (see also [15, 16]). Furthermore, such approach also suggests a possible way of understanding the cosmological constant issue [14. Hence, the study of one-loop generalized modified gravity is a natural step to be undertaken for the completion of such program, with the aim to better understand the role and the origin of quadratic corrections in the curvature. An alternative approach, which is in some sense alternative, has been proposed by Rueter and collaborators [17], see also the review paper [18], and [19], in which quantum gravity effects in astrophysics and cosmology are presented.

In the present paper, we will investigate in some detail a model described by a Lagrangian density which depends on geometric quadratic invariants. The quantization of quadratic models of gravity has been discussed in many papers, and in particular studied in detail on flat space in the seminal paper [20]. A preliminary discussion of a quadratic model based on one-loop on-shell results has been presented in [21].

Here we start with the classical Euclidean gravitational action

$$
I_{E}[g]=-\int d^{4} x \sqrt{g} F(R, P, Q)=-\int d^{4} x \sqrt{g}\left[M^{2} R-2 \alpha+b\left(R^{2}-3 P\right)+\beta G\right],
$$

where $b$ and $\beta$ are dimensionless parameters, $M^{2}$ a mass-squared parameter playing the role of gravitational coupling constant, and $\alpha$ a "cosmological constant" dimensional term. By $G$ we indicate the Gauss-Bonnet topological invariant which, in 4-dimensions, does not contribute to the classical field equations. For this reason, the action in (1.1) in classically equivalent to the so called Einstein-Weyl gravity, since the quadratic Weyl invariant $W$ and the Gauss-Bonnet invariant $G$ are related by

$$
G-W=\frac{2}{3}\left(R^{2}-3 P\right), \quad G=R^{2}-4 P+Q, \quad W=\frac{1}{3} R^{2}-2 P+Q,
$$

$P$ and $Q$ being

$$
P=R^{i j} R_{i j}, \quad Q=R^{i j r s} R_{i j r s}, \quad i, i, r, s=0,1,2,3 .
$$

As we already said above, at classical level the Gauss-Bonnet term does not play any role and could be dropped off but, as we shall see in the following, it do will play an important role at quantum level.

The action in the form (1.1) is quite useful in order to discuss the so called "critical gravity", which corresponds to a particular choice of the $b$ parameter. An extensive study of 4-dimensional 
critical gravity, in the presence of a negative cosmological constant, has been recently presented in Refs. 22, 23, 24, 25, where additional relevant references can be found.

It should be noted that one-loop Euclidean quantum gravity in a de Sitter background - as was fully exploited in [14] for the case of Einstein's gravity in the presence of a cosmological term-presents some peculiar aspects within the background field method. First, working with the Euclidean version $S(4)$, one is dealing with a geometric background associated with a compact manifold without a boundary. This means that the volume is finite and can be expressed as a function of the constant Ricci curvature, which may be chosen as background field. A second important remark is that, in order to discuss the one-loop renormalizability of the model, as well as the related renormalization group equations, one is forced to work with the off-shell one-loop effective action. As a consequence, the Landau gauge appears to be the most convenient one. Besides, the usual effective action calculated in this gauge coincides with the Vilkovisky-De Witt effective action (see, for example, [6]).

Such approach should be compared with the more traditional one, nicely reviewed in [26], where the Sakharov induced gravity approach [27] and its modern variants [28 have been discussed too. Conformal gravity has been discussed in 29]. The ghost absence issue for a very general gravitational quadratic model on Minkowski space-time has been recently investigated in [30. Furthermore, alternative approach is presented in [31.

Regarding to the choice of regularization, since we are dealing with non flat space-time, it is almost mandatory (or at least very convenient) to make use of a variant of the generalized zeta-function regularization [32, 33] (see also [34, 35, 36]), and the associated heat-kernel techniques [37, 38, 39]. In this way, one may evaluate the one-loop effective action and then study the possibility of stabilization of the de Sitter background by quantum effects.

The paper is organized as follows. Section II contains the evaluation of the quantum fluctuation operators relevant for the one-loop calculations to be carried out. In Section III, the off-shell one loop partition function is presented and the corresponding one loop renormalization is discussed. Finally, Section IV is devoted to conclusions.

\section{Quantum field fluctuations around maximally symmetric instan- tons}

In this Section we will discuss the one-loop quantization of the model in (1.1) on a maximally symmetric space (see, for instance [6]). To start with, we consider the Euclidean gravitational action in (1.1) and, for convenience, we separate linear and quadratic terms

$$
F(R, P, Q)=f(R)+b\left(R^{2}-3 P\right)+\beta G, \quad f(R)=M^{2} R-2 \alpha .
$$

The model admits a constant Ricci curvature solution $R_{0}$. In fact, the general equation for the existence of de Sitter solution [40, 41]

$$
\left[\left(\frac{1}{2} R \frac{\partial}{\partial R}+P \frac{\partial}{\partial P}+Q \frac{\partial}{\partial Q}-1\right) F(R, P, Q)\right]_{R=R_{0}}=0,
$$

is trivially satisfied, and reads

$$
f\left(R_{0}\right)-\frac{1}{2} R_{0} f^{\prime}\left(R_{0}\right)=0 \quad \Longrightarrow \quad R_{0}=\frac{4 \alpha}{M^{2}} .
$$

We are interested in studying quantum fluctuations around the Euclidean dS instanton $S^{4}$ with positive constant scalar curvature $R_{0}$. This is a maximally symmetric space having covariant conserved curvature tensors. Its metric may be written in the form

$$
d s_{E}^{2}=d \tau^{2}\left(1-H_{0}^{2} r^{2}\right)+\frac{d r^{2}}{\left(1-H_{0}^{2} r^{2}\right)}+r^{2} d S_{2}^{2},
$$


$d S^{2}$ being the metric of the two-dimensional sphere $S^{2}$ and $H_{0}$ the Hubble constant. The finite volume is given by

$$
V\left(S^{4}\right)=\frac{384 \pi^{2}}{R_{0}^{2}}, \quad R_{0}=12 H_{0}^{2}, \quad G_{0}=24 H_{0}^{4},
$$

while the Riemann and Ricci tensors are

$$
R_{i j r s}^{(0)}=\frac{R_{0}}{12}\left(g_{i r}^{(0)} g_{j s}^{(0)}-g_{i s}^{(0)} g_{j r}^{(0)}\right), \quad R_{i j}^{(0)}=\frac{R_{0}}{4} g_{i j}^{(0)} .
$$

Now let us consider small fluctuations around the maximally symmetric instanton. For the sake of completeness, we consider the general action discussed in [21], but linear in $P, Q$. Then, we shall restrict to the action (1.1), at the end of the computation. For simplicity, we also put $M^{2}=1$. When necessary the right units will be easily recovered by dimensional analysis.

We set

$$
g_{i j} \longrightarrow g_{i j}+h_{i j}, \quad g^{i j} \longrightarrow g^{i j}-h^{i j}+h^{i k} h_{k}^{j}+\mathcal{O}\left(h^{3}\right), \quad h=g^{i j} h_{i j},
$$

where from now on $g_{i j} \equiv g_{i j}^{(0)}$ is the metric of the maximally symmetric space and, as usual, indices are lowered and raised by means of such metric.

Up to second order in $h_{i j}$, one has

$$
\sqrt{g} \longrightarrow \sqrt{g}\left[1+\frac{1}{2} h+\frac{1}{8} h^{2}-\frac{1}{4} h_{i j} h^{i j}+\mathcal{O}\left(h^{3}\right)\right]
$$

and

$$
\begin{aligned}
R \sim & R_{0}-\frac{R_{0}}{4} h+\nabla_{i} \nabla_{j} h^{i j}-\Delta h \\
& +\frac{R_{0}}{4} h^{j k} h_{j k}-\frac{1}{4} \nabla_{i} h \nabla^{i} h-\frac{1}{4} \nabla_{k} h_{i j} \nabla^{k} h^{i j}+\nabla_{i} h_{k}^{i} \nabla_{j} h^{j k}-\frac{1}{2} \nabla_{j} h_{i k} \nabla^{i} h^{j k},
\end{aligned}
$$

where $\nabla_{k}$ represents the covariant derivative in the unperturbed metric $g_{i j}$. More complicated expressions are obtained for the other invariants $P, Q$, but for our aims it is not necessary to write them explicitly.

By performing a Taylor expansion of the Lagrangian around de Sitter metric, up to second order in $h_{i j}$, we get

$$
I_{E}[g] \sim-\int d^{4} x \sqrt{g}\left[F\left(R_{0}, P_{0}, Q_{0}\right)+\frac{h X}{2}+\mathcal{L}_{2}\right],
$$

where $\mathcal{L}_{2}$ represents the second-order contribution and $X$ vanishes when the de Sitter existence condition (2.2) is satisfied. For our particular model, $X=\left[f\left(R_{0}\right)-(1 / 2) R_{0} f^{\prime}\left(R_{0}\right)\right] / M^{2}$.

It is convenient to carry out the standard expansion of the tensor field $h_{i j}$ in irreducible components 14, namely

$$
h_{i j}=\hat{h}_{i j}+\nabla_{i} \xi_{j}+\nabla_{j} \xi_{i}+\nabla_{i} \nabla_{j} \sigma+\frac{1}{4} g_{i j}(h-\Delta \sigma),
$$

where $\sigma$ is the scalar component, while $\xi_{i}$ and $\hat{h}_{i j}$ are the vector and tensor components, with the following properties

$$
\nabla_{i} \xi^{i}=0, \quad \nabla_{i} \hat{h}^{i j}=0, \quad \hat{h}_{i}^{i}=0 .
$$

In terms of the irreducible components of the $h_{i j}$ field, the Lagrangian density, disregarding total derivatives, becomes

$$
\mathcal{L}_{2}=\mathcal{L}_{h h}+2 \mathcal{L}_{h \sigma}+\mathcal{L}_{\sigma \sigma}+\mathcal{L}_{V}+\mathcal{L}_{T},
$$


where $\mathcal{L}_{h h}, \mathcal{L}_{h \sigma}, \mathcal{L}_{\sigma \sigma}$ represent the scalar contribution (a $2 \times 2$ matrix), while $\mathcal{L}_{V}$ and $\mathcal{L}_{T}$ represent the vector and tensor contributions, respectively. One has

$$
\begin{gathered}
\mathcal{L}_{h h}=h\left[\frac{1}{32} F_{R R} R_{0}^{2}-\frac{1}{32} F_{R} R_{0}+\frac{X}{16}-\frac{3}{32} F_{R} \Delta+\frac{1}{16} F_{P} R_{0} \Delta+\frac{1}{16} F_{Q} R_{0} \Delta\right. \\
\left.+\frac{3}{16} F_{R R} R_{0} \Delta+\frac{3}{16} F_{P} \Delta^{2}+\frac{3}{16} F_{Q} \Delta^{2}+\frac{9}{32} F_{R R} \Delta^{2}\right] h, \\
\mathcal{L}_{h \sigma}=h\left[-\frac{1}{16} F_{R R} R_{0}^{2} \Delta+\frac{1}{16} F_{R} R_{0} \Delta-\frac{1}{8} F_{P} R_{0} \Delta^{2}\right. \\
-\frac{1}{8} F_{Q} R_{0} \Delta^{2}-\frac{3}{8} F_{R R} R_{0} \Delta^{2}+\frac{3}{16} F_{R} \Delta^{2} \\
\left.-\frac{3}{8} F_{P} \Delta^{3}-\frac{3}{8} F_{Q} \Delta^{3}-\frac{9}{16} F_{R R} \Delta^{3}\right] \sigma, \\
\mathcal{L}_{\sigma \sigma}=\sigma\left[\frac{1}{32} F_{R R} R_{0}^{2} \Delta^{2}-\frac{1}{16} X R_{0} \Delta-\frac{1}{32} F_{R} R_{0} \Delta^{2}-\frac{3}{16} X \Delta^{2}\right. \\
+\frac{1}{16} F_{P} R_{0} \Delta^{3}+\frac{1}{16} F_{Q} R_{0} \Delta^{3}+\frac{3}{16} F_{R R} R_{0} \Delta^{3}-\frac{3}{32} F_{R} \Delta^{3} \\
\left.+\frac{3}{16} F_{P} \Delta^{4}+\frac{3}{16} F_{Q} \Delta^{4}+\frac{9}{32} F_{R R} \Delta^{4}\right] \sigma, \\
\mathcal{L}_{V}=\xi^{k}\left[\frac{1}{8} R_{0} X+\frac{1}{2} X \Delta\right] \xi_{k}, \\
\mathcal{L}_{T}=h^{i j}\left[-\frac{1}{72} F_{P} R_{0}^{2}+\frac{1}{36} F_{Q} R_{0}^{2}-\frac{1}{24} F_{R} R_{0}-\frac{1}{4} X+\frac{1}{4} F_{R} \Delta\right. \\
\left.+\frac{1}{3} F_{Q} R_{0} \Delta+\frac{1}{4} F_{P} \Delta+F_{Q} \Delta^{2}\right] \hat{h}_{i j} .
\end{gathered}
$$

where $\Delta=g^{i j} \nabla_{i} \nabla_{j}$ is the Laplace-Beltrami operator in the unperturbed metric $g_{i j}$, which is a solution of the field equations, but only if $X=0$. We have written the above expansions around a maximally symmetric space, which in principle would not be a solution. This means, in other words, that the function $f(R)$ can be arbitrary. In the latter expression $F_{R}, F_{R R}$ represent the first and second derivatives of $F(R, P, Q)$ with respect to $R$ evaluated on de Sitter metric $g_{i j}$. And similarly for $F_{P}, F_{Q}$.

As is well known, invariance under diffeomorphisms renders the operator in the $(h, \sigma)$ sector not invertible. One needs a gauge fixing term and a corresponding ghost compensating term. Here we choose the harmonic gauge, that is

$$
\chi_{j}=-\nabla_{i} h_{j}^{i}-\frac{1}{2} \nabla_{j} h=0,
$$

and the gauge fixing term

$$
\mathcal{L}_{g f}=\frac{1}{2} \chi^{i} G_{i j} \chi^{j}, \quad G_{i j}=\gamma g_{i j} .
$$

The corresponding ghost Lagrangian reads [6]

$$
\mathcal{L}_{g h}=B^{i} G_{i k} \frac{\delta \chi^{k}}{\delta \varepsilon^{j}} C^{j}
$$


where $C_{k}$ and $B_{k}$ are the ghost and anti-ghost vector fields respectively, while $\delta \chi^{k}$ is the variation of the gauge condition due to an infinitesimal gauge transformation of the field. In this case, it reads

$$
\delta h_{i j}=\nabla_{i} \varepsilon_{j}+\nabla_{j} \varepsilon_{i} \quad \Longrightarrow \quad \frac{\delta \chi^{i}}{\delta \varepsilon^{j}}=g_{i j} \Delta+R_{i j} .
$$

Neglecting total derivatives, one has

$$
\mathcal{L}_{g h}=B^{k} \gamma\left(\Delta+\frac{R_{0}}{4}\right) C_{k} .
$$

In irreducible components one finally obtains

$$
\begin{gathered}
\mathcal{L}_{g f}=\frac{\gamma}{2}\left[\xi^{k}\left(\Delta+\frac{R_{0}}{4}\right)^{2} \xi_{k}+\frac{3 \rho}{8} h\left(\Delta+\frac{R_{0}}{3}\right) \Delta \sigma\right. \\
\left.-\frac{\rho^{2}}{16} h \Delta h-\frac{9}{16} \sigma\left(\Delta+\frac{R_{0}}{3}\right)^{2} \Delta \sigma\right] \\
\mathcal{L}_{g h}=\gamma\left[\hat{B}^{k}\left(\Delta+\frac{R_{0}}{4}\right) \hat{C}_{k}+\frac{\rho-3}{2} \hat{b}\left(\Delta-\frac{R_{0}}{\rho-3}\right) \Delta \hat{c}\right],
\end{gathered}
$$

where ghost irreducible components are defined by

$$
\begin{array}{ll}
C_{k}=\hat{C}_{k}+\nabla_{k} \hat{c}, & \nabla_{k} \hat{C}^{k}=0, \\
B_{k}=\hat{B}_{k}+\nabla_{k} \hat{b}, & \nabla_{k} \hat{B}^{k}=0 .
\end{array}
$$

\section{Off-shell one-loop effective action}

In order to compute the one-loop contributions to the effective action one has to consider the path integral for the bilinear part, $\mathcal{L}=\mathcal{L}_{2}+\mathcal{L}_{g f}+\mathcal{L}_{g h}$, of the total Lagrangian and take into account the Jacobian due to the change of variables with respect to the original ones. In this way, one gets [14, 6]

$$
\begin{aligned}
Z^{(1)}= & \left(\operatorname{det} G_{i j}\right)^{-1 / 2} \int D\left[h_{i j}\right] D\left[C_{k}\right] D\left[B^{k}\right] \exp \left(-\int d^{4} x \sqrt{g} \mathcal{L}\right) \\
= & \left(\operatorname{det} G_{i j}\right)^{-1 / 2} \operatorname{det} J_{1}^{-1} \operatorname{det} J_{2}^{1 / 2} \\
& \times \int D[h] D\left[\hat{h}_{i j}\right] D\left[\xi^{j}\right] D[\sigma] D\left[\hat{C}_{k}\right] D\left[\hat{B}^{k}\right] D[c] D[b] \exp \left(-\int d^{4} x \sqrt{g} \mathcal{L}\right),
\end{aligned}
$$

where $J_{1}$ and $J_{2}$ are the Jacobians coming from the change of variables in the ghost and tensor sectors, respectively [14]. They read

$$
J_{1}=\Delta_{0}, \quad J_{2}=\left(\Delta_{1}+\frac{R_{0}}{4}\right)\left(\Delta_{0}+\frac{R_{0}}{3}\right) \Delta_{0},
$$

and the determinant of the operator $G_{i j}$ is trivial in this case. Here and in the following $\Delta_{0}, \Delta_{1}, \Delta_{2}$, represent the Laplacian acting on scalars, vectors and tensors, respectively.

Due to the presence of curvature, the Euclidean gravitational action is not bounded from below, because arbitrary negative contributions can be induced on $R$ by conformal rescaling of the metric. For this reason we have also used the Hawking prescription of integrating over imaginary scalar fields. Furthermore, the problem of the presence of additional zero modes introduced by the decomposition (2.11) can be treated by making use of the method presented in Ref. [14.

Now, for the action (1.1) a straightforward computation leads to the off-shell one-loop contribution to the "partition function". In the Landau gauge, $\rho=1, \gamma \rightarrow \infty$, with $X=R_{0} / 2-2 \alpha / M^{2}$, we get

$$
\Gamma_{\text {off-shell }}=I_{E}(g)+\Gamma_{o f f-s h e l l}^{(1)}, \quad I_{E}(g)=96 \pi^{2}\left(\frac{2 M^{2}}{R_{0}}+b\right)+64 \pi^{2} \beta,
$$




$$
\begin{aligned}
\Gamma_{\text {off-shell }}^{(1)}= & \sum_{i} \frac{1}{2} \log \operatorname{det} \frac{L_{i}}{\mu^{2}}=\frac{1}{2} \log \operatorname{det}\left(\frac{1}{\mu^{2}}\left[-\Delta_{0}-\frac{2 \alpha}{M^{2}}\right]\right) \\
& \quad-\frac{1}{2} \log \operatorname{det}\left(\frac{1}{\mu^{2}}\left[-\Delta_{1}-\frac{R_{0}}{4}\right]\right)-\frac{1}{2} \log \operatorname{det}\left(\frac{1}{\mu^{2}}\left[-\Delta_{0}-\frac{R_{0}}{2}\right]\right) \\
& +\frac{1}{2} \log \operatorname{det}\left(\frac{1}{\mu^{2}}\left[-\Delta_{2}-Y_{+}\right]\right)+\frac{1}{2} \log \operatorname{det}\left(\frac{1}{\mu^{2}}\left[-\Delta_{2}-Y_{-}\right]\right)
\end{aligned}
$$

where

$$
Y_{ \pm}=\frac{1}{12}\left(-3 R_{0}-\frac{2 M^{2}}{b} \pm \frac{1}{b} \sqrt{96 b \alpha+4 M^{4}-20 b M^{2} R_{0}+b^{2} R_{0}^{2}}\right) .
$$

As usual, an arbitrary renormalization parameter $1 / \mu^{2}$ has been introduced for dimensional reasons. As expected, the parameter $\beta$ does not appear in the latter expression, since the Gauss-Bonnet invariant does not give contributions to the field equations, but it gives a constant contribution to the classical action, which will actually play an important role in the renormalization procedure.

\subsection{On-shell one-loop effective action}

As is well known, the on-shell effective action does not have to depend on the gauge and, in fact, setting $X=0$, that is $M^{2} R_{0}-4 \alpha=0$, we get

$$
\begin{aligned}
\Gamma_{\text {on-shell }} & =96 \pi^{2}\left(\frac{2 M^{2}}{R_{0}}+b\right)+64 \pi^{2} \beta-\frac{1}{2} \log \operatorname{det}\left(\frac{1}{\mu^{2}}\left[-\Delta_{1}-\frac{R_{0}}{4}\right]\right) \\
& +\frac{1}{2} \log \operatorname{det}\left(\frac{1}{\mu^{2}}\left[-\Delta_{2}+\frac{R_{0}}{6}\right]\right)+\frac{1}{2} \log \operatorname{det}\left(\frac{1}{\mu^{2}}\left[-\Delta_{2}+\frac{R_{0}}{3}+\frac{M^{2}}{3 b}\right]\right) .
\end{aligned}
$$

The above expression is only formal, and one needs regularization. For the moment, let us imagine to be dealing with the finite part of such an effective action.

We observe that there exists a "critical" value for $b$ for which all spin excitations become "massless". In fact, choosing

$$
b=b_{\text {crit }}=-\frac{2 M^{2}}{R_{0}}=-\frac{M^{4}}{2 \alpha},
$$

the effective action simplifies to

$$
\Gamma_{\text {crit }}=64 \pi^{2} \beta-\frac{1}{2} \log \operatorname{det}\left(\frac{1}{\mu^{2}}\left[-\Delta_{1}-\frac{R_{0}}{4}\right]\right)+\log \operatorname{det}\left(\frac{1}{\mu^{2}}\left[-\Delta_{2}+\frac{R_{0}}{6}\right]\right) .
$$

In contrast to the AdS case, in Euclidean dS space $R_{0}>0$, and so $b_{\text {crit }}<0$.

The stability of the dS solution can be investigated by looking at the spectra of the Laplace-type operators and it then follows that all eingenvalues are non-negative as in general relativity, with the possible presence of a zero mode [14, 9]. As a consequence, dS background space is stable, in agreement with the classical analysis presented in [40, 41].

But what about the stability of the critical values with respect to renormalization? According to the background field method one should work at the off-shell level. Nevertheless, one may try an on-shell, one-loop renormalization, by observing that $\beta$ might become a "bare" constant, and its redefinition may contain all counterterms necessary in order to cancel the on-shell one-loop divergences coming from the functional determinants. In general, using a variant of the zeta function regularization procedure [21], at one-loop level one has

$$
\Gamma(\mu, \varepsilon)=I_{E}(\mu, \epsilon)-\frac{1}{2} \sum_{i}\left[\frac{\zeta\left(0 \mid L_{i}\right)}{\varepsilon}+\zeta\left(0 \mid L_{i}\right) \log \mu^{2}+\zeta^{\prime}\left(0 \mid L_{i}\right)\right],
$$


where the summation is over all Laplace-type operators appearing in the one-loop contribution to the action.

For the critical gravity in (3.8), we have $L_{1}=-\Delta_{1}-R_{0} / 4$ and $L_{2}=-\Delta_{2}+R_{0} / 6$, thus

$$
\begin{aligned}
\Gamma(\mu, \varepsilon)=64 \pi^{2}\left(\frac{\beta_{0}}{\varepsilon}\right. & +\beta(\mu))+\frac{1}{2 \varepsilon}\left[\zeta\left(0 \mid L_{1}\right)-2 \zeta\left(0 \mid L_{2}\right)\right] \\
& +\log \mu\left[\zeta\left(0 \mid L_{1}\right)-2 \zeta\left(0 \mid L_{2}\right)\right]+\frac{1}{2} \zeta^{\prime}\left(0 \mid L_{1}\right)-\zeta^{\prime}\left(0 \mid L_{2}\right),
\end{aligned}
$$

where $\beta_{0}$ is the bare coupling constant and $\beta(\mu)$ the running one. Making a suitable choice for $\beta_{0}$, one has the renormalized on-shell effective critical action

$$
\Gamma_{\text {crit }}(\mu)=64 \pi^{2} \beta(\mu)+\log \mu\left[\zeta\left(0 \mid L_{1}\right)-2 \zeta\left(0 \mid L_{2}\right)\right]+\frac{1}{2} \zeta^{\prime}\left(0 \mid L_{1}\right)-\zeta^{\prime}\left(0 \mid L_{2}\right) .
$$

The eigenvalues of the Laplace type operators on $S O(4)$ are well known and in this way it is possible to compute the zeta-functions appearing in the expression above explicitly. In particular, $\zeta\left(0 \mid L_{1}\right)=$ $-191 / 30$ and $\zeta\left(0 \mid L_{2}\right)=89 / 9$, while $\zeta^{\prime}\left(0 \mid L_{1}\right)$ and $\zeta^{\prime}\left(0 \mid L_{2}\right)$ are computable expressions independent on $\mu$. The usual imposition

$$
\mu \frac{d \Gamma(\mu)}{d \mu}=0
$$

gives rise to the renormalization group equation for critical gravity, in the form

$$
\mu \frac{d \beta(\mu)}{d \mu}=2 \zeta\left(0 \mid L_{2}\right)-\zeta\left(0 \mid L_{1}\right) \sim 26>0 .
$$

This is the only running coupling constant and, thus, on-shell critical gravity seems to be stable at the one-loop level. But this is not really conclusive since, strictly, the issue of criticality depends on the on-shell expression.

\subsection{Off-shell one-loop renormalization}

As far as the off-shell one-loop renormalization is concerned, the situation is completely different with respect to the previous one and apparently there is no room for the notion of criticality.

Again, the starting point is the equation in (3.9), but now the classical action contains all the bare quantities, which generate the counterterms for absorbing the one-loop divergencies. It reads

$$
\begin{aligned}
I_{E}(\mu, \epsilon) & =384 \pi^{2}\left[\frac{M^{2}(\mu, \varepsilon)}{R_{0}}-\frac{2 \alpha(\mu, \varepsilon)}{R_{0}^{2}}+\frac{b(\mu, \varepsilon)}{4}+\frac{\beta(\mu, \varepsilon)}{6}\right] \\
& =384 \pi^{2}\left[\frac{M^{2}(\mu)}{R_{0}}-\frac{2 \alpha(\mu)}{R_{0}^{2}}+\frac{b(\mu)}{4}+\frac{\beta(\mu)}{6}\right]+\frac{1}{\varepsilon}\left[\frac{A_{1}}{R_{0}}+\frac{B_{1}}{R_{0}^{2}}+C_{1}\right],
\end{aligned}
$$

where we have separated the finite and divergent parts of the coupling constants by means of suitable finite quantities $A_{1}, B_{1}, C_{1}$ independent of $R_{0}$. On the other hand, a direct computation shows that

$$
\sum_{i} \zeta\left(0 \mid L_{i}\right)=\left[\frac{A(\mu)}{R_{0}}+\frac{B(\mu)}{R_{0}^{2}}+C(\mu)\right]
$$

where $L_{i}$ are all Laplace-type operators in (3.4) and $A(\mu), B(\mu), C(\mu)$ are finite functions depending on the renormalized running coupling constants $M^{2}(\mu), \alpha(\mu), b(\mu)$. They read

$$
A(\mu)=\frac{8 \alpha(\mu)}{M^{2}(\mu)}, \quad B(\mu)=\frac{20 M^{4}(\mu)}{3 b^{2}(\mu)}+\frac{80 \alpha(\mu)}{b(\mu)}+\frac{48 \alpha^{2}(\mu)}{M^{4}(\mu)}, \quad C(\mu)=\frac{1763}{90} .
$$


The model is one-loop renormalizable since all one-loop divergences can actually be absorbed by an appropriate choice of $A_{1}, B_{1}, C_{1}$. The finite, renormalized one-loop effective action reads

$$
\Gamma(\mu)=384 \pi^{2}\left[\frac{M^{2}(\mu)}{R_{0}}-\frac{2 \alpha(\mu)}{R_{0}^{2}}+\frac{b(\mu)}{4}\right]+\log \mu\left[\frac{A(\mu)}{R_{0}}+\frac{B(\mu)}{R_{0}^{2}}+C(\mu)\right]+Z,
$$

where we have dropped the parameter $\beta(\mu)$ because here it does not play any role, and we have set $Z=-\frac{1}{2} \sum_{i} \zeta^{\prime}\left(0 \mid L_{i}\right)$. This is the finite part of the functional determinant which does not depend on $\mu$ and in principle can be explicitly evaluated.

As above, the one-loop renormalization group equations can be obtained by means of (3.12). To this aim it is convenient to introduce the dimensionless variable $\rho=\log \frac{\mu}{\mu_{0}}, \mu_{0}$ being a reference low energy scale. ¿From (3.12) we obtain the three differential equations

$$
\left\{\begin{array}{l}
\frac{d b}{d \rho}=c_{b}, \\
\frac{d M^{2}}{d \rho}=\frac{\alpha}{48 \pi^{2} M^{2}}, \\
\frac{d \alpha}{d \rho}=-\frac{1}{192 \pi^{2} b^{2}}\left(12 \frac{\alpha^{2} b^{2}}{M^{4}}+20 \alpha b+\frac{5}{3} M^{4}\right),
\end{array} \quad c_{b}=\frac{1763}{8640 \pi^{2}},\right.
$$

where all coupling constants are functions of $\rho$. Solving the system of differential equations above, we finally get

$$
\left\{\begin{array} { l } 
{ b ( \rho ) = c _ { b } \rho + c _ { 0 } , } \\
{ M ^ { 2 } ( \rho ) = c _ { 1 } ( \rho + c _ { 0 } ) ^ { p _ { 1 } - p _ { 2 } } [ ( \rho + c _ { 0 } ) ^ { 1 0 p _ { 2 } } + c _ { 2 } ] ^ { 1 / 5 } , } \\
{ \alpha ( \rho ) = 4 8 \pi ^ { 2 } \frac { M ^ { 4 } ( \rho ) } { ( \rho + c _ { 0 } ) } [ p _ { 1 } - p _ { 2 } + \frac { 2 p _ { 2 } } { 1 + c _ { 2 } ( \rho + c _ { 0 } ) ^ { - 1 0 p _ { 2 } } } ] , }
\end{array} \quad \left\{\begin{array}{l}
p_{1}=\frac{863}{17630} \\
p_{2}=\frac{\sqrt{474769}}{17630}
\end{array}\right.\right.
$$

The integration constants $c_{0}, c_{1}, c_{2}$ depend on the initial conditions and we assume all of them to be non negative. Moreover, to simplify the discussion, from now on we shall take $c_{2}=0$. With this assumption we get

$$
M^{2}(\rho)=c_{1}\left(\rho+c_{0}\right)^{p}, \quad \alpha(\rho)=48 \pi^{2} p \frac{M^{4}(\rho)}{\rho+c_{0}}, \quad \quad p=p_{1}+p_{2} \sim 0.09,
$$

and the one-loop, running, gravitational coupling constant reads

$$
G(\rho)=\frac{1}{16 \pi M^{2}(\rho)}=\frac{1}{16 \pi c_{1}\left(\rho+c_{0}\right)^{p}},
$$

while the one-loop, running, cosmological constant is

$$
\Lambda(\rho)=\frac{\alpha(\rho)}{M^{2}(\rho)}=48 \pi^{2} p c_{1}\left(\rho+c_{0}\right)^{p-1} .
$$

As a result, there is no Landau pole, and at large energy $\mu \gg \mu_{0}$ or equivalently $\rho \rightarrow \infty$, one has that both $G(\rho)$ and $\Lambda(\rho)$ go to zero. This property is the analogue on de Sitter space of the well known gravitational asymptotic freedom for quadratic gravity [42, 43, 44, 45, 46].

Furthermore, we may assume general relativity to be valid at low energy, that is $\mu \sim \mu_{0}$ or equivalently $\rho \sim 0$, then

$$
\left.G(\rho)\right|_{\rho \rightarrow 0}=G_{N} \quad \Longrightarrow \quad c_{1}=\frac{c_{0}^{-p}}{16 \pi G_{N}}, \quad M^{2}(\rho)=\frac{1}{16 \pi G_{N}}\left(1+\frac{\rho}{c_{0}}\right)^{p}
$$

$G_{N}=G(0)$ being the Newton constant.

To conclude this section, we write down the effective field equation given by

$$
\frac{\partial \Gamma}{\partial R_{0}}=0 \text {. }
$$


The solution can be written in the implicit form

$$
R_{0}=\frac{1}{1-\frac{A \rho}{384 \pi^{2} M^{2}}}\left[\frac{4 \alpha}{M^{2}}-\frac{B \rho}{192 \pi^{2} M^{2}}+\frac{R_{0}^{3}}{384 \pi^{2} M^{2}} \frac{\partial Z}{\partial R_{0}}\right] .
$$

This is a quite complicated expression in the unknown variable $R_{0}$. Of course, at low energy $\rho \sim 0$, $R_{0} \ll M^{2}$, one gets the classical solution

$$
R_{0} \sim \frac{4 \alpha(0)}{M_{P}^{2}(0)}=4 \Lambda(0)
$$

but in principle other regimes can be studied.

\section{Conclusions}

In this paper, the Einstein gravity plus a quadratic gravitational Weyl term has been investigated by computing the corresponding one-loop quantum corrections by means of the background field method. As a classical background we have considered the de Sitter one for its potentially very important physical applications. The one-loop calculation has been performed in the Euclidean sector, where the classical background is the compact manifold $S^{4}$. In the calculation, due to the fact that we are working with a non flat background, we are forced to make use of (a variant of) zeta-function regularization. In the presence of this compact curved manifold, the one-loop effective action, and also the ensuing one-loop renormalization group equations, have been computed and carefully investigated. On shell, the associated quadratic critical gravity has been discussed. In order to investigate the role of the one-loop corrections, we have to consider the off-shell one-loop effective action and so, to get rid of the gauge dependence, the Landau gauge has been used. In this way the critical conditions are lost, in general. In fact, also in the simplified case we have considered $\left(c_{2}=0\right)$, the critical ratio $\frac{M^{4}(\rho)}{2 \alpha(\rho)} \sim\left(\rho+c_{0}\right)$, which is not equal to $b(\rho)$. This means that the critical gravity condition are not stable under the one-loop renormalization flow. As a consequence, one might doubt about its relevance at least when the background is the compact manifold $S^{4}$. In fact, in the anti de Sitter case (AdS) the situation could be completely different, because the Euclidean counterpart of AdS is the non compact hyperbolic manifold $H^{4}$.

In conclusion, it should also be interesting to repeat this one-loop calculation in alternative extended gravity models, for example, in the so-called $f(T)$ gravity models, which depend on geometric invariants built up by using torsion field (for details see [47] and the references therein).

\section{Acknowledgments}

We would like to thank L. Vanzo and S.D. Odintsov for valuable suggestions. Part of this work was done at the Kobajashi-Maskawa Institute in Nagoya, and S.Z. and E.E. would like to thank all the members of Center for Theoretical Studies for very kind hospitality. E.E. was partly supported by MICINN (Spain), grant PR2011-0128 and project FIS2010-15640, by the CPAN Consolider Ingenio Project, and by AGAUR (Generalitat de Catalunya), contract 2009SGR-994.

\section{References}

[1] S. Nojiri and S. D. Odintsov, eConf C0602061, 06 (2006) [Int. J. Geom. Meth. Mod. Phys. 4, 115 (2007)] arXiv:hep-th/0601213].

[2] S. Nojiri and S. D. Odintsov, Phys. Rept. 505, 59 (2011) [arXiv:1011.0544 [gr-qc]]. 
[3] T. P. Sotiriou and V. Faraoni, Rev. Mod. Phys. 82, 451 (2010) arXiv:0805.1726 [gr-qc]].

[4] A. De Felice and S. Tsujikawa, Living Rev. Rel. 13, 3 (2010) [arXiv:1002.4928 [gr-qc]].

[5] A. A. Starobinsky, Phys. Lett. B 91, 99 (1980).

[6] I.L. Buchbinder, S.D. Odintsov and I.L. Shapiro, Effective action in quantum gravity, IOP Publishing, Bristol, 1992.

[7] G. Cognola, E. Elizalde, S. Nojiri, S. D. Odintsov, L. Sebastiani and S. Zerbini, Phys. Rev. D 77, 046009 (2008) arXiv:0712.4017 [hep-th]].

[8] G. Cognola, E. Elizalde, S. Nojiri, S. D. Odintsov and S. Zerbini, JCAP 0502, 010 (2005);

[9] G. Cognola and S. Zerbini, J. Phys. A 39, 6245 (2006) arXiv:hep-th/0511233; G. Cognola, L. Sebastiani and S. Zerbini, to appear in proceedings MG12, arXiv:1006.1586 [gr-qc] (2009).

[10] G. Cognola, E. Elizalde, S. Nojiri, S. D. Odintsov and S. Zerbini, Eur. Phys. J. C 64, 483 (2009) arXiv:0905.0543 [gr-qc]].

[11] G. Cognola, E. Elizalde, S. Nojiri and S. D. Odintsov, Open Astron. J. 3, 20 (2010) arXiv:0909.2747 [gr-qc]].

[12] G.W. Gibbons and M.J. Perry, Nucl. Phys. B146 (1978) 90.

[13] S.M. Christensen and M.J. Duff, Nucl. Phys. B170 (1980) 480.

[14] E.S. Fradkin and A.A. Tseytlin, Nucl. Phys. B234 (1984) 472.

[15] S.D. Odintsov, Europhys. Lett. 10 (1989) 287; Theor. Math. Phys.82 (1990) 66; T.R. Taylor and G. Veneziano, Nucl. Phys. B345 (1990) 210.

[16] D. V. Vassilevich, Int. J. Mod. Phys. A 8, 1637 (1993).

[17] O. Lauscher and M. Reuter, Phys. Rev. D 65, 025013 (2001) hep-th/0108040.

[18] M. Niedermaier and M. Reuter, Living Rev. Rel. 9, 5 (2006).

[19] M. Reuter and H. Weyer, JCAP 0412, 001 (2004) hep-th/0410119; M. Reuter and H. Weyer, Phys. Rev. D 70, 124028 (2004) hep-th/0410117); E. Bentivegna, A. Bonanno and M. Reuter, JCAP 0401, 001 (2004) astro-ph/0303150|. M. Reuter and F. Saueressig, JCAP 0509, 012 (2005) [hep-th/0507167]; A. Bonanno and M. Reuter, Int. J. Mod. Phys. D 13, 107 (2004) astro-ph/0210472; A. Bonanno and M. Reuter, Phys. Rev. D 65, 043508 (2002) hep-th/0106133.

[20] K. S. Stelle, Phys. Rev. D 16, 953 (1977).

[21] G. Cognola and S. Zerbini, "One-loop F(R,P,Q) gravity in de Sitter universe," J. Phys. A: Math. Theor. 45374014 (2012).

[22] H. Lu and C. N. Pope, Phys. Rev. Lett. 106, 181302 (2011) arXiv:1101.1971 [hep-th]]; H. Lu, Y. Pang and C. N. Pope, Phys. Rev. D 84, 064001 (2011) arXiv:1106.4657 [hep-th]].

[23] H. Lu, C. N. Pope, E. Sezgin and L. Wulff, JHEP 1110, 131 (2011) [arXiv:1107.2480 [hep-th]].

[24] H. Lu, Y. Pang, C. N. Pope and J. F. Vazquez-Poritz, Phys. Rev. D 86, 044011 (2012) arXiv:1204.1062 [hep-th]]. 
[25] K. Hirochi and S. Nojiri, arXiv:1203.3301 [hep-th]

[26] M. Visser, Mod. Phys. Lett. A 17, 977 (2002) gr-qc/0204062.

[27] A.D. Sakharov, Sov. Phys. Dokl. 121040 (1968).

[28] V. P. Frolov and D. V. Fursaev, Phys. Rev. D 56, 2212 (1997) hep-th/9703178;

V. P. Frolov, D. V. Fursaev and A. I. Zelnikov, Nucl. Phys. B 486, 339 (1997) hep-th/9607104.

[29] G. de Berredo-Peixoto and I. L. Shapiro, Phys. Rev. D 70, 044024 (2004) hep-th/0307030.

[30] T. Biswas, E. Gerwick, T. Koivisto and A. Mazumdar, Phys. Rev. Lett. 108, 031101 (2012) arXiv:1110.5249 [gr-qc]].

[31] A. Codello and R. Percacci, Phys. Rev. Lett. 97, 221301 (2006) hep-th/0607128.

A. Codello, R. Percacci and C. Rahmede, Int. J. Mod. Phys. A 23, 143 (2008) arXiv:0705.1769 [hep-th]].

P. F. Machado and R. Percacci, Phys. Rev. D 80, 024020 (2009) [arXiv:0904.2510 [hep-th]].

A. Codello, R. Percacci and C. Rahmede, Annals Phys. 324, 414 (2009) arXiv:0805.2909 [hepth]].

A. Bonanno, A. Contillo and R. Percacci, Class. Quant. Grav. 28, 145026 (2011) arXiv:1006.0192 [gr-qc]].

[32] J. S. Dowker and R. Critchley, Phys. Rev. D 13, 3224 (1976).

[33] S. W. Hawking, Commun. Math. Phys. 55, 133 (17).

[34] E. Elizalde, S.D. Odintsov, A. Romeo, A.A. Bytsenko and S. Zerbini. Zeta regularization techniques with applications, World Scientific, 1994; E. Elizalde, Ten physical applications of spectral zeta functions, 2nd Ed., Springer, Berlin, 2012.

[35] A.A. Bytsenko, G. Cognola, L. Vanzo and S. Zerbini, Phys. Reports. 269 (1996)1.

[36] K. Kirsten, Spectral Functions in Mathematics and Physics, Chapamn and Hall, CRC, London (2000).

[37] B.S. DeWitt. The Dynamical Theory of Groups and Fields. Gordon and Breach, New York (1965).

[38] R.T. Seeley. Am. Math. Soc. Prog. Pure Math. 10, 172 (1967).

[39] D. V. Vassilevich, Phys. Rept. 388, 279 (2003) hep-th/0306138.

[40] G. Cognola, M. Gastaldi and S. Zerbini, Int. J. Theor. Phys. 47, 898 (2008) gr-qc/0701138.

[41] G. Cognola and S. Zerbini, Int. J. Theor. Phys. 47 (2008) 3186 [arXiv:0802.3967 [hep-th]].

[42] E. S. Fradkin and G. A. Vilkovisky, Phys. Lett. B 77, 262 (1978).

[43] E. Tomboulis, Phys. Lett. B 97, 77 (1980).

[44] I. G. Avramidi and A. O. Barvinsky, Phys. Lett. B 159, 269 (1985).

[45] I. G.'Avramidi, Lectures Notes in Physics M 64 1-149, 2000, Spinger.

[46] G. Narain and R. Anishetty, Phys. Lett. B 711, 128 (2012) arXiv:1109.3981 [hep-th]].

[47] K. Bamba, R. Myrzakulov, S. Nojiri and S. D. Odintsov, Phys. Rev. D 85, 104036 (2012) arXiv:1202.4057 [gr-qc]]. 